\title{
PROCEDIMIENTO PARA EL APUNTAMIENTO DE UNA ANTENA TERRESTRE A UN SATELITE GEOESTACIONARIO BSS (BROADCAST SATELLITE SERVICE)
}

MIRANDA, Carlos A.; SCHLESINGER, Paola L.; VALDEZ, Alberto D.; CHIOZZA, Juan A.; MIRANDA, Carlos V. (*)

\section{RESUMEN:}

La instalación de satélites en el espacio constituye uno de los mayores avances de la tecnología del siglo pasado, que permitió la comunicación entre lugares del mundo muy distantes. Los satélites geoestacionarios son utilizados en la actualidad para fines científicos, tecnológicos, meteorológicos, militares, y servicios de transmisión de televisión, internet y telefonía. Este trabajo reúne las consideraciones generales para realizar el apuntamiento de una antena parabólica hacia un satélite geoestacionario proveedor de servicios de televisión digital, BSS (Broadcast Satellite Service), mediante el uso de expresiones utilizadas para el cálculo de la orientación de antenas satelitales. Y de esta forma apuntar las mismas a partir de la posición orbital del satélite geoestacionario y de la posición geográfica de la antena terrestre. E1 servicio de BSS tiene mucha similitud con el servicio fijo FSS (Fixed $\mathrm{Sa}$ tellite Service) ya que las antenas parabólicas de recepción de los usuarios permanecen fijas, por lo que el método es de aplicación también para este servicio.

Palabras claves: elevación; frecuencia; satélite; traspondedor, parabólica.

\section{INTRODUCCIÓN}

Uno de los resultados más importantes obtenidos a partir de los programas espaciales es la tecnología de los satélites artificiales. La llegada de estos complejos desarrollos electrónicos ha modificado visiblemente la forma de vida de la mayor parte de la población del mundo, y quizá de toda ella, aunque sea en forma indirecta.

Gracias a esta tecnología conocemos con mayor precisión los recursos naturales de la tierra y los fenómenos meteorológicos; las distancias entre ciudades y los países se han acortado y ahora pueden intercambiar todo tipo de información casi instantáneamente; y más allá de las capas atmosféricas podemos observar y comprender mejor el

$\left({ }^{*}\right)$ Facultad de Ciencias Exactas y Naturales y Agrimensura, Departamento de Ingeniería, UNNE, Corrientes CP 3400 , Argentina. E-mail: cmiranda@exa.unne.edu.ar 
Universo. En general, todos los satélites artificiales funcionan bajo el mismo principio y constan de varias partes comunes, independiente de su objetivo, en órbita alrededor de la tierra. Si bien hay algunas diferencias fundamentales entre ellos, todos necesitan, por ejemplo de celdas solares para alimentarse de energía, antenas para transmitir su información a ciertos puntos del planeta y recibir instrucciones o cualquier tipo de señal, además de medios de propulsión para corregir su órbita, posición u orientación con respecto a la Tierra.

Los satélites integran una gran familia y parte de ella la constituyen los que están avocados específicamente a los servicios de comunicaciones; dentro de estos últimos existen algunas variantes, pero los geoestacionarios son los más importantes y de mayor uso en la actualidad [1].

Este trabajo busca sistematizar el método para el apuntamiento de una antena satelital utilizada para el servicio de distribución directa de TV, y aplicable a todo enlace entre una antena terrena y un satélite geoestacionario.

\section{DEFINICIONES}

\section{1 Órbita geoestacionaria}

El satélite en órbita geoestacionaria se desplaza en el mismo sentido de rotación que la Tierra, completa una vuelta cada 24 horas, para mantenerse en órbita debe estar a aproximadamente a $36.000 \mathrm{~km}$ de altura sobre el nivel del mar con una velocidad constante de $3.705 \mathrm{~m} / \mathrm{s}$, siguiendo una órbita circular alrededor del planeta de aproximadamente de $265.000 \mathrm{~km}$ y en el plano del ecuador. Éstas características lo hacen ideal para satélites de telecomunicación ya que el servicio está disponible en forma permanente.

\section{2 Ángulos de elevación y azimut}

Para orientar la antena de una estación terrena en la dirección correcta hacia donde está el satélite con el que se desea comunicarse, se definen los ángulos de elevación y azimut. Estos ángulos son medidos tomando como referencia la línea sobre la antena que tiene máxima ganancia, en una antena parabólica es el eje del plato parabólico. Este eje contiene al vértice y al foco.



Fig. 1. Angulo de elevacion $\theta$

El ángulo de elevación $\theta$ se define como el ángulo formado entre el plano horizontal local y la línea vista entre la estación terrena y el satélite como se muestra en la figura 1 .

$\mathrm{El}$ ángulo azimutal $\varphi$, es el ángulo medido en el sentido de las manecillas del reloj entre la línea que une a la estación terrena con el norte geográfico y la proyección horizontal local de la línea de máxima radiación de la antena, que debe apuntar en la dirección hacia el satélite, como se especifica en la figura 2. 
Tanto el valor del ángulo de elevación $\theta$ como el azimut $\varphi$ dependen de las coordenadas geográficas de la estación terrena y de la posición orbital del satélite de comunicación.

\subsection{Rango}

Es la distancia que hay entre una estación terrena y un satélite geoestacionario, denominada $\mathrm{d}$ en el gráfico y puede ser calculada a partir de la geometría de la figura 3.

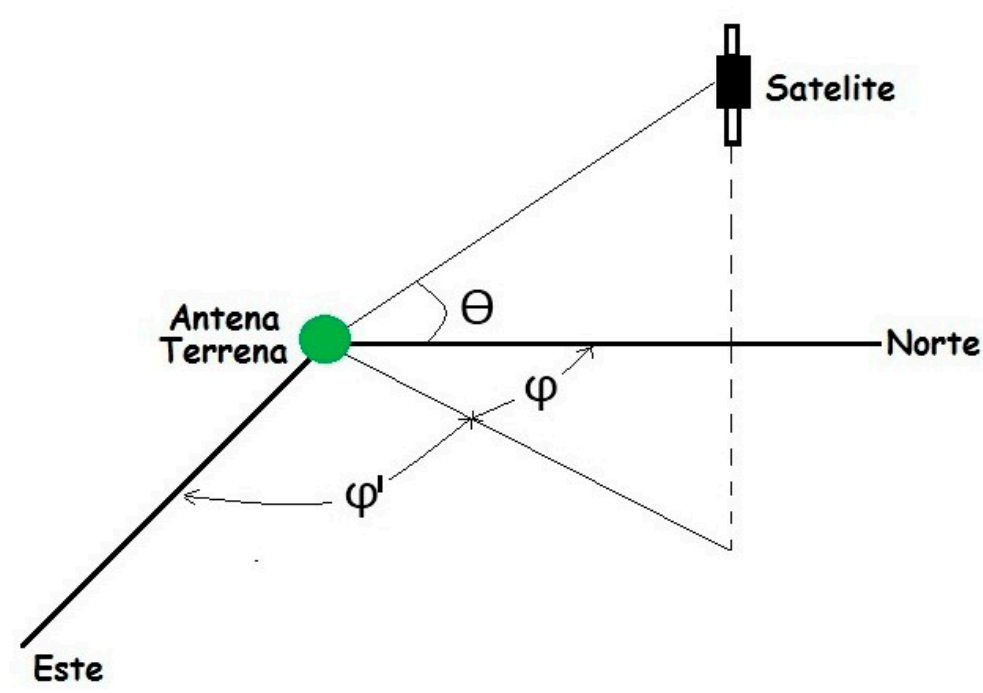

Figura 2. Ángulo azimutal $\varphi$ y complementario $\varphi^{\prime}$

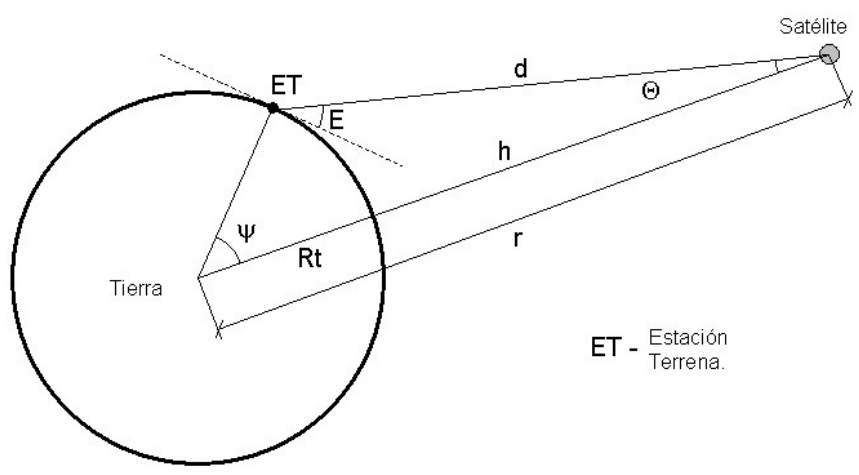

Figura 3 Geometria para el cáculo del rango o distancia d.

\section{MATERIALES Y MÉTODOS}

\subsection{Bandas de Frecuencias}

La capacidad de tráfico de un satélite está limitada por el ancho de banda y la potencia disponible en el mismo. La Unión Internacional de Telecomunicaciones (UIT) ha asignado para estos las bandas de VHF, UHF (sub banda L y S) y SHF (sub banda $\mathrm{C}, \mathrm{X}, \mathrm{Ku}$ y $\mathrm{Ka}$ ) que son empleadas por satélites civiles y militares para diferentes tipos de servicios. Los satélites comerciales funcionan en las bandas $\mathrm{C}, \mathrm{Ku}$ y $\mathrm{Ka}$. La banda Ku (Kurz - unten band) es una porción del espectro electromagnético en el rango de las microondas que va desde 12 a $18 \mathrm{GHz}$. Esta se usa principalmente en las comunicaciones satelitales, siendo la televi- 
sión uno de los principales servicios. Esta banda se divide en segmentos que cambian por regiones geográficas. La mayor parte del continente americano se encuentra dentro de la Región 2 de la UIT; habiendo asignado la banda de frecuencia ascendente de 12,5 a $12,75 \mathrm{GHz}$ y descendente de 11,45 a 11,7 GHz. Presenta un ancho de banda para los enlaces de subida y bajada de 500 $\mathrm{MHz}$ para los servicios de radiodifusión por satélite que es una categoría especial en la que las señales transmitidas son recibidas directamente en los hogares (Direct Broadcast Service / Satellite DBS).

\subsection{Antenas con reflectores pasivos}

Para las comunicaciones satelitales se requieren antenas con ganancia y directividad muy altas. Las antenas que proporcionan estos dos requerimientos son las antenas reflectoras parabólicas multihaz, estas tienen dos componentes: un reflector parabólico (elemento pasivo) y un mecanismo de alimentación (elemento activo). $\mathrm{El}$ reflector es un dispositivo que refleja la energía irradiada por el mecanismo de alimentación. Este tipo de reflector permite modificar los diagramas de radiación de un alimentador, mejorando sus características. Las antenas reflectoras y discos parabólicos son las más importantes en la banda de microondas $(1 \mathrm{GHz}$ a $300 \mathrm{GHz})$. Las ventajas que poseen son de grandes ganancias y uso adecuado en varias frecuencias. [2] Las ondas irradiadas desde una fuente ubicada en el foco se reflejan en la parábola y configuran un frente de onda plano. En general la ganancia máxima Gmáx de una antena de apertura está dada por:

$$
G \max =\eta \frac{4 \pi A}{\lambda^{2}}
$$

En donde $\eta$ es la eficiencia de la antena, A es el área física de su apertura (limitada por la trayectoria cerrada de su borde) y $\lambda$ es la longitud de onda a la frecuencia de trabajo. Al producto de la eficiencia por el área física se le denomina apertura efectiva. Los platos parabólicos son iluminados desde su foco geométrico por antenas de banda ancha y la eficiencia del conjunto normalmente es del orden del $55 \%$ o mayor hasta un 70 o $75 \%$. El ancho del lóbulo principal de radiación de un plato parabólico definido como el ángulo entre los puntos de media potencia, se pueden calcular en forma aproximada como:

$$
\theta_{-3 d B}=\frac{75 \lambda}{D}
$$

Donde $\mathrm{D}$ es el diámetro del plato. Como la dimensión de una antena parabólica se especifica en términos de su diámetro expresamos la ecuacion 5 en función de $\mathrm{D}$, así:

$$
\begin{gathered}
A=\pi \cdot \text { radio }^{2}=\frac{\pi \cdot D^{2}}{4} \\
G \max =\eta \frac{4 \pi \cdot \pi \cdot D^{2}}{\lambda^{2} 4}
\end{gathered}
$$

Entonces tenemos:

$$
G \max =\eta\left(\frac{\pi D}{\lambda}\right)^{2} \approx 65,8(D f)^{2}
$$

Con D en metros y f en GHz. Es usual expresar la ganancia en decibeles con rela- 
ción a la ganancia unitaria de la antena isotrópica $(\mathrm{dBi})$, tenemos:

$$
[G]_{d B i}=10 \log G
$$

Para calcular la ganancia en otras direcciones, que no sea el máximo dentro del ancho del haz principal, entre los dos puntos de media potencia, podemos tomar para una dirección desviada $\alpha$ grados con relación al eje principal de radiación, por medio de la siguiente expresión en decibeles

$$
[G \alpha]_{d B i}=[G \max ]_{d B i}-12\left(\frac{\alpha}{\theta_{-3 A B i}}\right)[\mathrm{dBi}]
$$

Para el rango de $0^{\circ} \leq \alpha \leq \frac{\theta_{-3 d B}}{2}$

En donde

$$
\theta_{-3 d b}=\frac{75 \lambda}{D}
$$

Para evitar la sombra que produce el elemento activo, se utilizan antenas parabólicas con alimentación descentrada denominadas OFF-SET [3] como se observa en la Figura 4. Este tipo de antena es utilizadas en las redes privadas de comunicación y en los modernos equipos de recepción de TV digital directa en los hogares, en la banda Ku. [4]

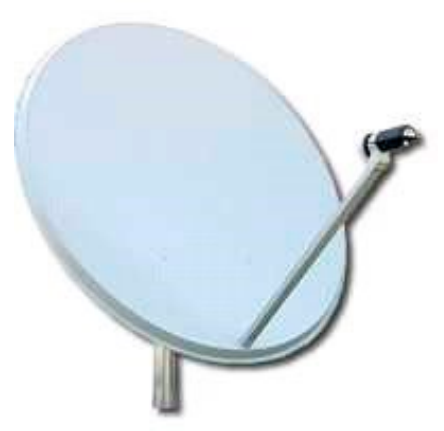

Figura 4. Antena parabólica OFF-SET

\subsection{Alimentador}

Los alimentadores de las estaciones terrenas para uso comercial generalmente son del tipo de antenas de corneta o bocina, ya sea con apertura rectangular (corneta piramidal) o circular (corneta cónica), como se observa en la Figura 5 y actúan iluminando adecuadamente al reflector, permitiendo discriminar señales en las bandas de transmisión y recepción, separar y combinar polarizaciones si el sistema es de doble polarización, y aportar señales de error de apuntamiento en los sistemas de rastreo. [5] [6]. La corneta corrugada con ensanchamiento de $90^{\circ}$ usada como alimentador es la de mayor utilización de las estaciones para recepción directa de TV. Las frecuencias de operación del amplificador de bajo ruido LNB (Low Noise Block), incorporado en el iluminador en la banda Ku del servicio BSS (Broadcast Satellite Service) son las siguientes: de entrada banda baja de 10,7 $-11,7 \mathrm{GHz}$, frecuencia del oscilador local 9,75 GHz y frecuencia de salida banda baja $950-1700(\mathrm{MHz})$. [7] [8]

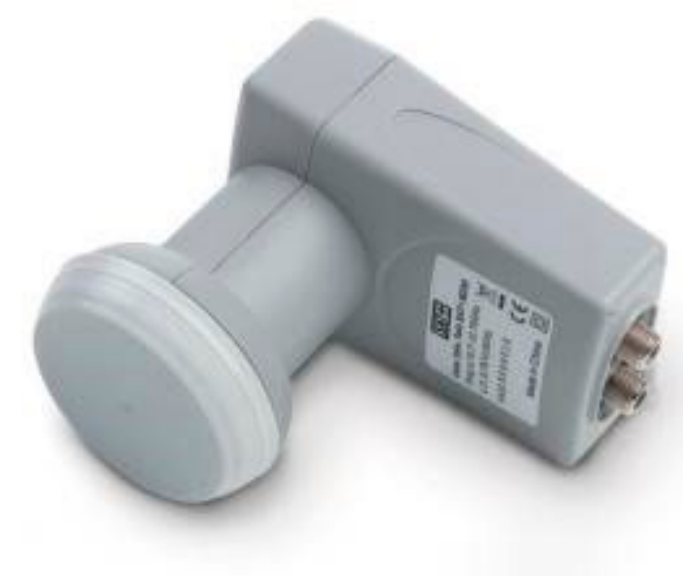

Figura $5 L N B$ 
Los contornos de la potencia isotrópica radiada equivalente, PIRE, es la potencia entregada a la antena del satélite, enlace de bajada, por la ganancia de esta en cierta dirección, como se observa en la figura 6, en donde el punto central, marcado con una cruz, es donde va dirigido el PIRE. Alrededor de dicha trayectoria se encuentran varios contornos de PIRE constante, y el valor de este va disminuyendo conforme abarca mayor área y limitan zonas de potencia en la recepción, se miden en $\mathrm{dBW}$, y permite de esta forma calcular el diámetro de la antena parabólica de recepción [9].

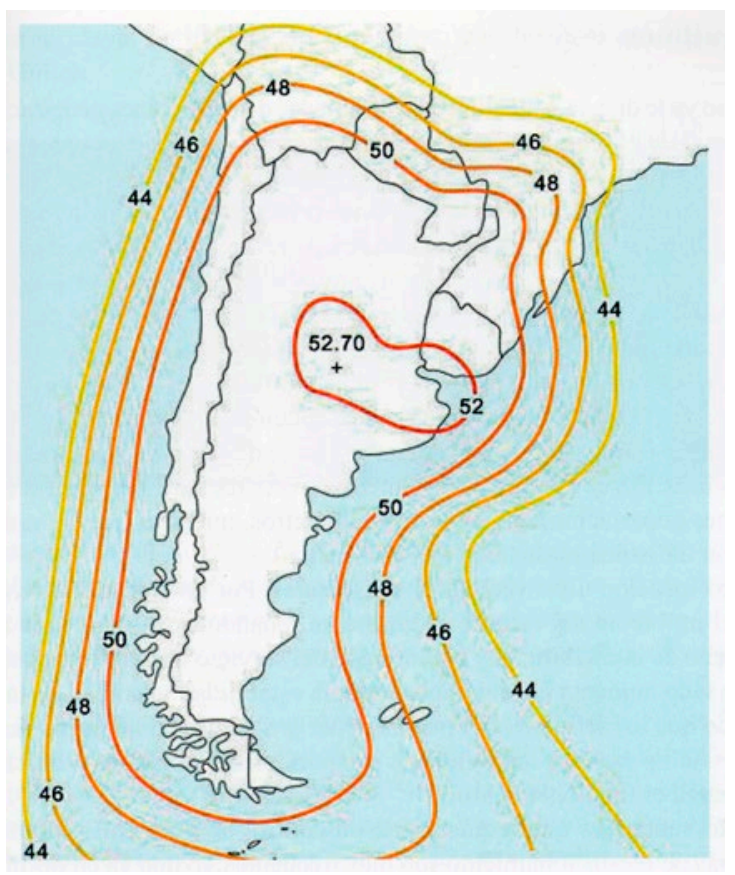

Figura 6 Huellas de un satelite sobre la República Argentina

\subsection{Expresiones para el Cálculo del ángu-} lo de elevación, azimutal y distancia entre la estación terrena y el satélite geoestacionario.

Las expresiones de cálculo para el ángulo de elevación es:

$$
\theta=\tan ^{-1}\left\{\frac{(\cos l) \cdot(\cos \Delta L)-\frac{\mathrm{R}_{t}}{h}}{\operatorname{sen}\left[\cos ^{-1}(\cos l \cdot \cos \Delta L)\right]}\right\}
$$

\section{Donde}

1 = latitud geográfica de la estación terrena

$\Delta \mathrm{L}=$ longitud geográfica orbital del satélite - longitud geográfica de la estación terrena

$\mathrm{Rt}=$ radio de la Tierra $=6.378 \mathrm{~km}$

$\mathrm{h}=$ radio de la órbita $=42.164 \mathrm{~km}$

Y el ángulo azimutal $\varphi$, dependiendo de la localización de la estación terrena con relación al satélite, se halla a partir de:

- $\varphi=180^{\circ}-\varphi^{\prime}$ Estación en el hemisferio norte y al oeste del satélite.

- $\varphi=180^{\circ}+\varphi^{\prime}$ Estación en el hemisferio norte y al este del satélite.

- $\varphi=\varphi^{\prime}$ Estación en el hemisferio sur y al oeste del satélite.

- $\varphi=360^{\circ}-\varphi^{\prime}$ Estación en el hemisferio sur y al este del satélite.

Donde

$$
\phi^{\prime}=\tan ^{-1}\left\{\frac{.(\operatorname{sen} \Delta L)}{\operatorname{sen} l \cdot \cos \Delta L}\right\}=\operatorname{tg}^{-1}\left[\frac{\tan \Delta L}{\operatorname{sen} l}\right]
$$

Las expresiones analíticas anteriores suponen que la latitud del satélite es exactamente $0^{\circ}$.

Cálculo del ángulo $\Psi$, de la distancia d entre la estación terrena y el satélite geoestacionario, de acuerdo a la figura 3, El ángulo de elevación $\theta, \mathrm{E}$ para el gráfico, se calcula previamente por la ecuación 9, con $\mathrm{h}$ igual a $35.786 \mathrm{Km}$, Rt igual a $6.378 \mathrm{Km}$ y $\Psi$ lo obtenemos a partir de: 


$$
\Psi=\cos ^{-1}[(\cos l) \cdot(\cos \Delta L)]
$$

Así:

$$
d=35.786 \sqrt{1,4199-0,4199 \cdot \cos \Psi}(\mathrm{Km})
$$

IV. PROCEDIMIENTO PARA EL APUNTAMIENTO DE UNA ANTENA TERRESTRE A UN SATÉLITE GEOESTACIONARIO

1) Determinar las coordenadas geográficas de la estación terrena.

2) Ubicar el Norte geográfico terrestre.

3) Seleccionar el satélite geoestacionario proveedor del servicio, su posición orbital y cobertura.

4) Cálculo del ángulo de elevación (expresión 9)

5) Cálculo del ángulo azimutal (expresión 10)

6) Cálculo del rango (expresión 11 y

7) Instalar la antena satelital y conectar el alimentador al decodificador Satellite Receiver.

8) Conectar la segunda salida del alimentador a un Analizador de Espectro de ancho de banda adecuado.

9) Posicionar el LNB para la correcta polarización.

10) Desplazar la antena hasta los ángulos determinado en 4 y 5, hasta máxima indicación en el analizador de espectro

4.1 Apuntamiento de una antena terrestre parabólica tipo off-set de 1,22 metros de diámetro al satélite geoestacionario, BSS, Direc TV 4S, Banda Ku.
1) Coordenadas geográficas de la estación terrena ubicada en el Campus Universitario de la FaCENA:

Latitud Sur 27²7'57,6”. Longitud oeste $58^{\circ} 47^{\prime} 07,1^{\prime}$. Determinadas con el Sistema de Posicionamiento Global GPS (Global Positioning System) marca Garmin Nuvi.

2) Se ubica el Norte geográfico por medio de la brújula marca Recta modelo DP6.

3) Longitud 101,20 Oeste. Cobertura sobre América Latina. Fuente: LingSat, http://www.lyngsat.info
4) Ángulo de elevación $\theta=33.9^{\circ}$
5) Ángulo acimutal $\varphi=63,82^{\circ}$
6) Rango d=38.289 Km
7) Antena parabólica tipo OFF-SET de 1,22 metros de diámetro. Iluminador y LNB modelo SH P02, marca SHARP, 40 $\mathrm{dB}$, receptor satelital marca THOMPSON CONSUMER ELECTRONICS.

8) Analizador de Espectro GW-INSTEK $100 \mathrm{KHz} 2.7 \mathrm{GHz}$

9) Se rotó el LNB para polarización correcta obteniéndose máxima indicación.

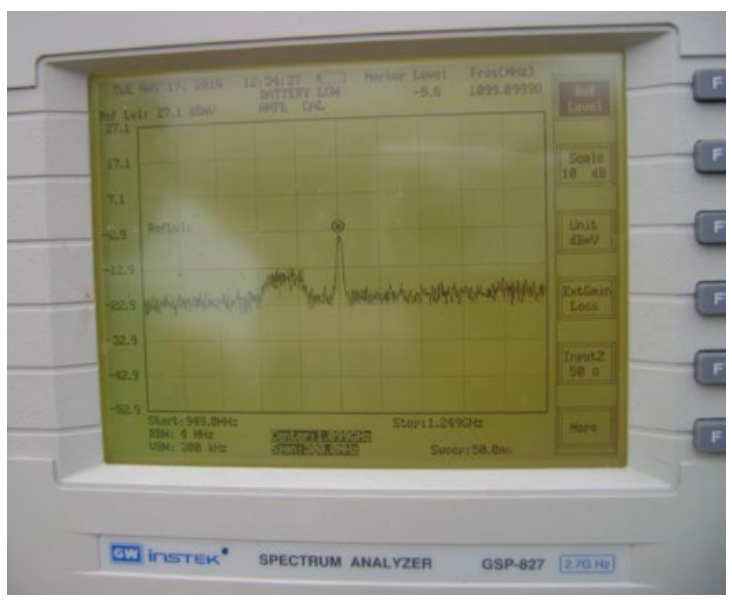

Figura 7. Señal observada y registrada en el analizador de espectro. 




Figura 8. Grupo de alumnos realizando la tarea de apuntamiento de la antena

10) La señal del satélite geoestacionario DirecTV 4S, se identificó a una frecuencia de 1,099 $\mathrm{GHz}$, con un nivel de $-5,6 \mathrm{~dB} \mu \mathrm{V}$. Figura 7.

E1 trabajo de apuntamiento fue realizado por el grupo de RNI con alumnos del último año de la Carrera Ingeniería en Electrónica de la FaCENA (Figura 8).

\subsection{Análisis del nivel medido}

$\mathrm{E} 1$ resultado de $-5,6 \mathrm{~dB} \mu \mathrm{V}$ es producto de la potencia efectiva radiada por la antena satelital, PIRE, correspondiente al contorno del área de cobertura, atenuada 184,9 dB debido a la perdida por propagación en el espacio libre y elevado su nivel en 20,7 dB correspondiente a la ganancia de la antena terrena y $40 \mathrm{~dB}$ del LNB. Los niveles de potencia que se manejan en estos enlaces, dada las distancia de $38.289 \mathrm{Km}$ entre las antenas, calculado a partir de sus posiciones geográficas, son extremadamente bajos; por ello el apuntamiento de la antena terrena debe hacerse con extremada precisión.

\section{CONCLUSION}

La evolución tecnológica del servicio satelital de comunicaciones BSS, fue acompañada con el aumento de las frecuencias de utilizacion, desde la banda $\mathrm{C}$ para retransmitir $\mathrm{TV}$, de 3,9 a $6 \mathrm{GHz}$ a la de utilizacion actual de la banda $\mathrm{Ku}$ y de ocupacion de la banda Ka, 20 a $30 \mathrm{GHz}$. El aumento exponencial de servicios y transmisiones satelitales proximas a la saturación en las bandas de frecuencia asignadas obliga a una identificacion precisa del satélite proveedor del servicio y un apuntamiento con un alto grado de precisión para identificar y obtener la máxima señal del satélite operador del servicio. 


\section{LÍNEAS FUTURAS}

El Grupo de Estudio de Radiaciones No Ionizantes del Departamento de Ingeniería de la Facultad de Ciencias Exactas de la Universidad Nacional del Nordeste, coautores del presente trabajo, continuará identificando y midiendo los niveles de las RNI, a las que se están incorporando y contribuyendo las señales satelitales. Para ello y conjuntamente con alumnos de último año de la Carrera Ingeniería en Electrónica se está incorporando el desarrollo de un dispositivo para la orientación de antenas en forma automática a partir de la posición orbital de los satélites geoestacionarios a apuntar, y con las antenas adecuadas para medir los niveles de señal con que llegan a la superficie terrestre y contribuyen a los niveles de RNI existentes.

\section{VII- REFERENCIAS}

[1] Neri Vela, Rodolfo "Comunicaciones por satélite", Editorial THOMSON.

[2] Bava, J. A. y Sanz, A. J. "Microondas y recepción satelital", Edicion del CEILP.

[3] Balanis, C.A. "Antenna Theory, Analisis and Desing”, 2o edicion, Wiley, 1997.

[4] Sistemas VSAT y Estaciones Terrenas, Suplemento No3 del Manual sobre Telecomunicaciones por Satelite (servicio Fijo) UIT.Ginebra 1993
[5] Conversores LNB (Low Noise Block-Down)Televes

[6] http://www.lyngsat-maps.com/

[7] Bava, J. A. "Microondas y Recepción Satelital. pp.37-41”, Edición del CELP. La Plata, Argentina, 1.999.

[8] Aznar, Ángel C. “Antenas”. Alfaomega. México, 2004.

[9] Cabrera, Ricardo "Satelites". Eudeba, Ciudad Autonoma de Buenos Aires, 2015. 